\title{
Artefactual Allotyping Related to DNA Source, Concentration and the Number of PCR Cycles
}

\author{
Brian B. Cohen, Valerie A. Anderson and \\ Karen Gillespie \\ University of St. Andrews, Bute Medical \\ Building, St. Andrews Fife, KY16 9TS, UK
}

KEYWORDS: PCR, artefacts, allotypes

Microsatellites amplified by the polymerase chain reaction are used extensively for determining genetic linkage, allelic imbalance, and mismatch repair deficiencies in tumours. Sometimes unexpected results are obtained; an allele known to be present is not detected or, conversely, new alleles appear to be produced. The former may be explained by a mutation in the primer sequence and the allele can be detected by using a redesigned primer [4]. The latter phenomenon is usually ascribed to defects in mismatch repair genes [5], however changes in allele size may arise from other causes and seem to be especially prevalent when the DNA has been prepared from archival wax-embedded tissues.

Microsatellites, particularly dinucleotide repeats, are notorious for producing PCR products which include shadow bands ("laddering") on analytical polyacrylamide gels, making the true alleles difficult to discern [2]. Attempts to reduce the laddering usually involve lowering the number of cycles in the PCR and/or the primer concentrations.

In the course of our studies on pedigree analysis and allelic imbalance, we have noted that reducing the number of PCR cycles often leads to a change in the apparent allotype, because at higher cycle numbers shadow bands become "alleles". However, low cycle numbers can fail to produce detectable PCR products, especially when the DNA is derived from archival wax blocks (probably due to the poor state of the DNA or to the presence of inhibitors).

An experiment was therefore undertaken to examine formally the relationship between amount of template, cycle number, and extent of laddering. Figure 1 shows the results of a PCR using the same DNA template at different concentrations, prepared from fresh and waxembedded placental tissue, and for varying the number of PCR cycles. The true allele status is only obvious in some of the 20 and 30 cycle tracks and not in any of those run for 40 or 50 cycles. It is noteworthy that a very high concentration of "wax" DNA template did give a true picture after 30 cycles whereas almost all of the other readable wax DNA/cycle combinations generated completely erroneous "alleles". Indeed, track 2 of the 50 cycle set would almost certainly have been scored as a case of allelic instability had the experiment been a normal versus tumour DNA comparison. Likewise tracks 2 and 3 of the 40 cycle set could be scored as allelic imbalance. Conversely at higher cycle numbers, "fresh" DNA at all concentrations produced extensive laddering.

Figure 2 shows a short exposure autoradiograph of an allotyping analysis with fresh DNA carried out using 20 and 35 cycles. Again, clear results were obtained with the lower cycle number.

This study confirms the existence of artefacts that have been recorded on many occasions but, to our knowledge, never formally assessed. The above demonstration is intended to act as a 


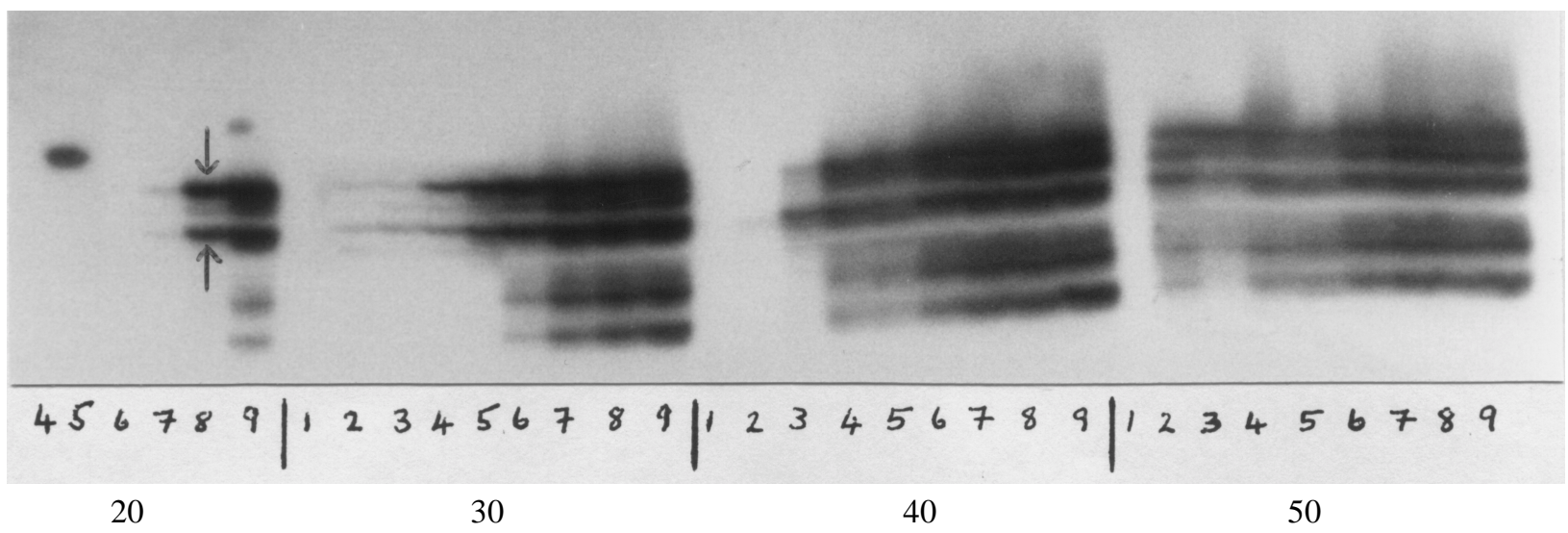

Fig. 1. Effect on allotyping of PCR cycle number, DNA source and concentration $1-2 \mathrm{~g}$ of human placenta was fixed in formalin and wax embedded; $10 \mu$ sections were cut and DNA extracted using a commercial kit (Nuclear Biosciences). DNA was prepared from fresh placenta in the same way. PCR mixes (50 $\mu$ l) were set up using primers for the D1S3644 locus allowing 20, 30, 40 and 50 cycles. Lanes 1-4, 5 fold increasing concentrations of "wax" DNA. Lanes 5-9 PCR mixes containing 2ng, 20ng, 200ng, $2 \mu \mathrm{g}$ and 10 $\mu \mathrm{g}$ fresh DNA respectively. The PCR products were denatured and separated on an $8 \%$ sequencing gel, passively transferred to nylon membrane and probed with a ${ }^{32} \mathrm{P}$-labelled $(\mathrm{CA})_{22}$ oligonucleotide [1]. The true alleles are indicated with arrows.

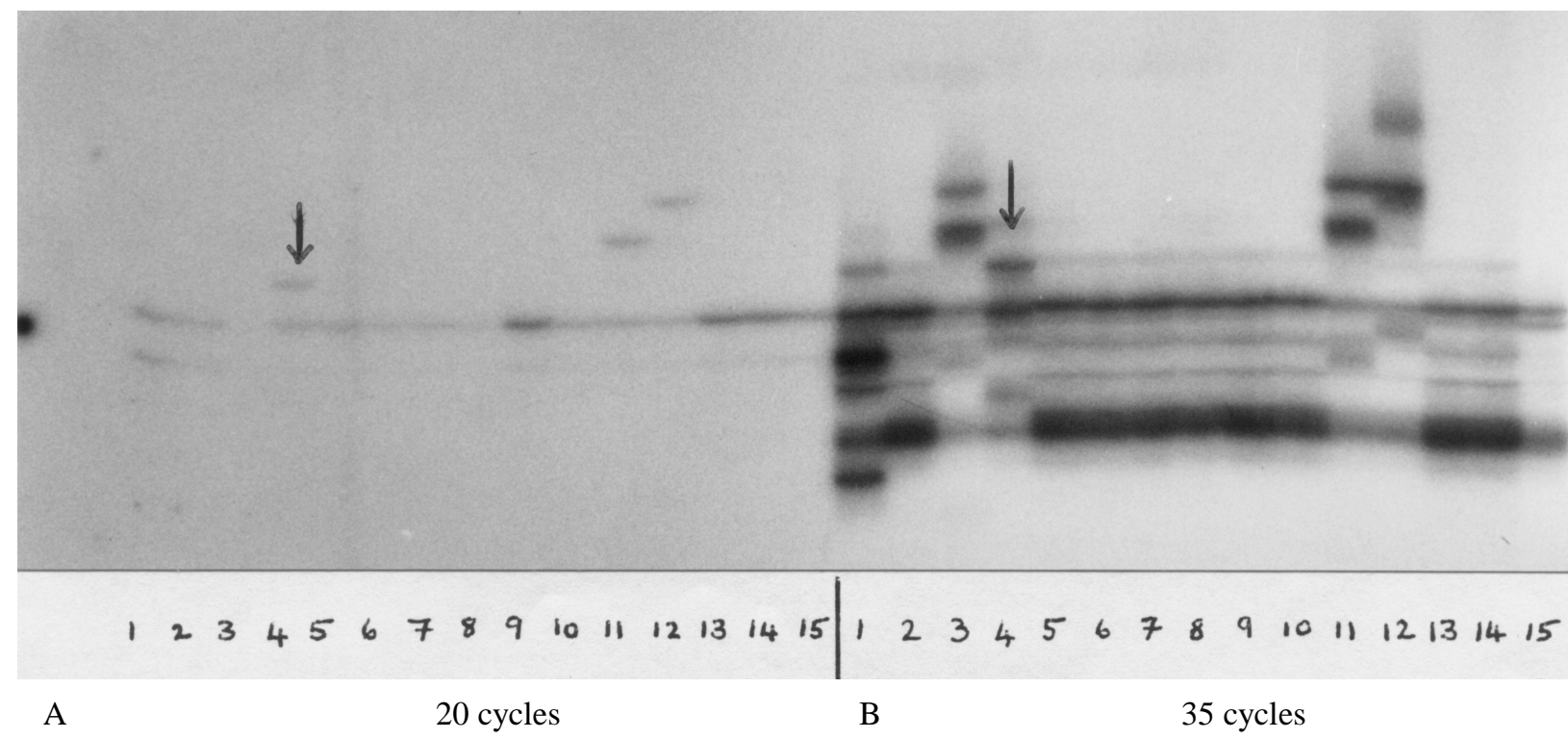

Fig. 2. Allotyping analysis using 20 and 35 cycles. DNA prepared from fresh blood of 15 related individuals was amplified using primers for the D17S1322 locus. A, 20 cycles, B, 35 cycles. Note individual 4 shows correct alleles with both numbers of cycles, but in general the 35 cycle analysis is difficult to interpret and would give erroneous results.

caveat. It is common practice to measure the template concentration when DNA is prepared from fresh tissue but this is rarely done when it is derived from archival material, nor do most workers use less than 30 cycles in the PCR. We suggest that, whenever alleles "disappear" or "new" or "unexpected" alleles appear, the PCR be repeated with a range of template concentrations at lower cycle numbers before final allotypes are assigned. 


\section{Acknowledgement}

The authors gratefully acknowledge support from the Scottish Office Department of Health (Chief Scientist Office).

\section{References}

[1] Cohen, B.B., Wallace, M.R. and Crichton, D.N. A comparison of procedures for analysing microsatellite (CA) repeat polymorphisms. Mol. and Cell. Probes 6, (1992) 439-442.
[2] Hauge, Y. and Litt, M. A study of the origin of shadow bands seen when typing dinucleotide repeat polymorphisms by the PCR. Hum. Mol. Genet. 2(4), (1993) 411-415.

[3] Hammel, S., Burger, J., Rameckers, J. et al. Amplifications 14(1), (1996) 5-9.

[4] Koorey, D.J., Bishop, G.A. and McCaughan W. Allele non-amplification: a source of confusion in linkage studies employing microsatellite polymorphisms. Hum. Mol. Genet. 2(3) (1993) 289-291.

[5] Lothe, R.A. Microsatellite instability in human solid tumours. Mol. Med. Today 3, (1997) 61-68. 


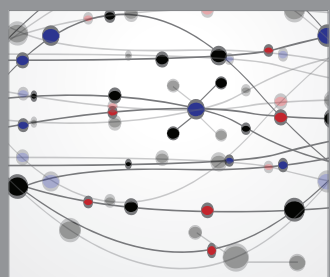

The Scientific World Journal
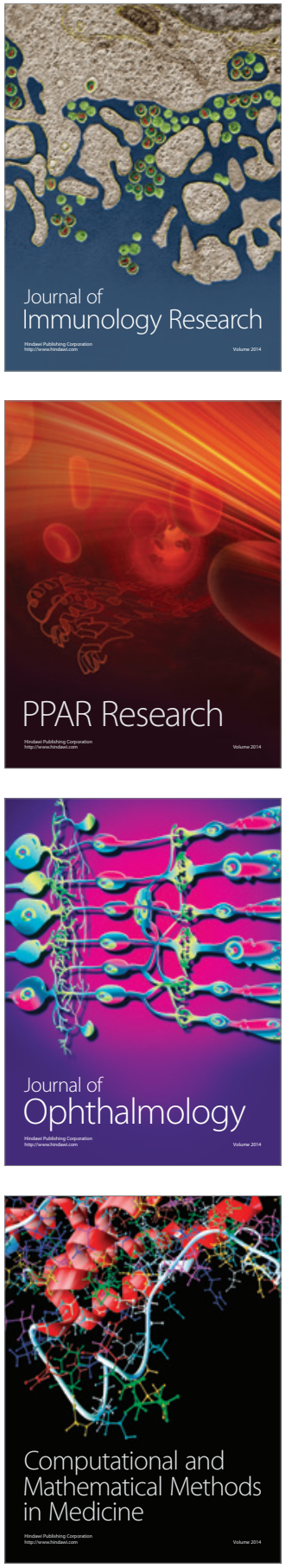

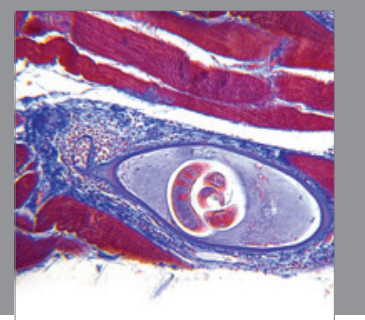

Gastroenterology

Research and Practice
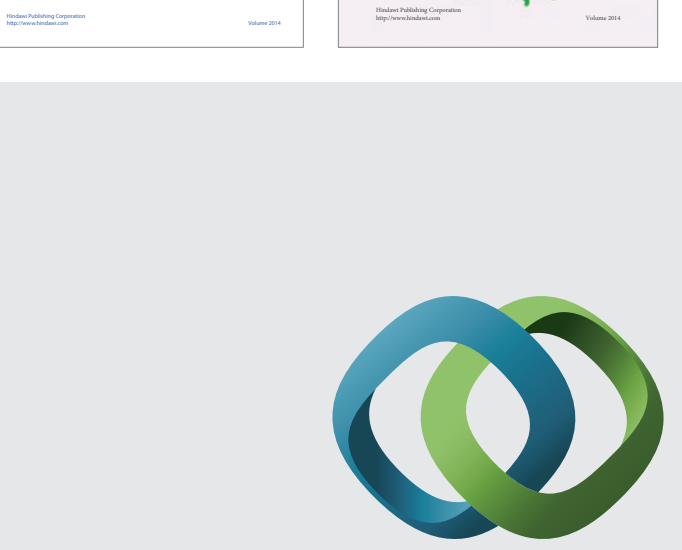

\section{Hindawi}

Submit your manuscripts at

http://www.hindawi.com
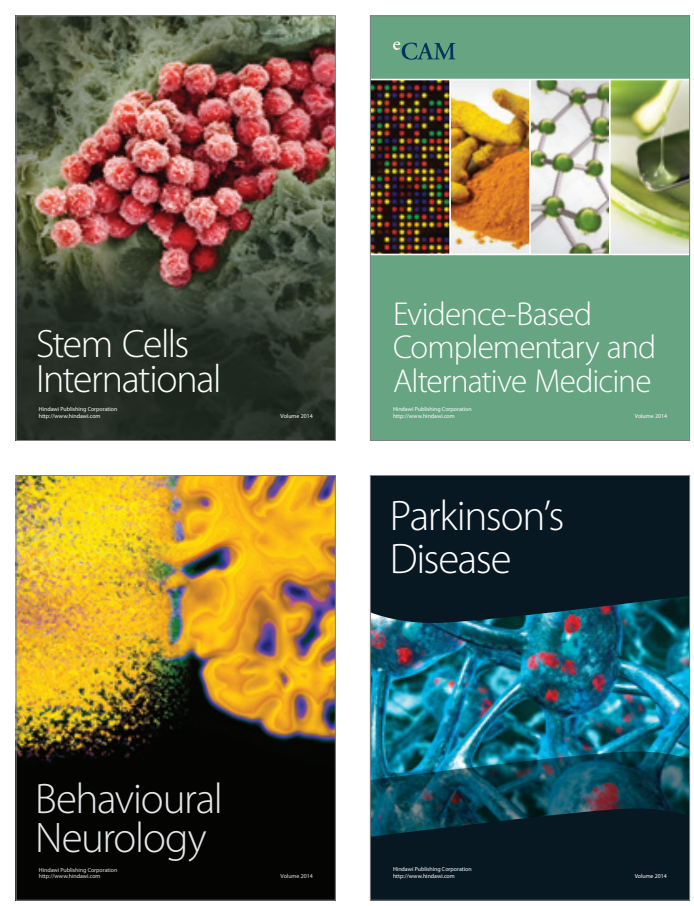

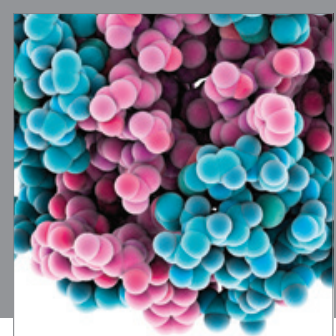

Journal of
Diabetes Research

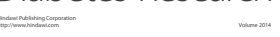

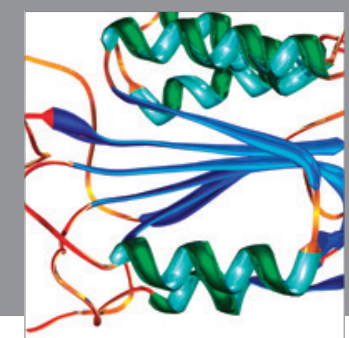

Disease Markers
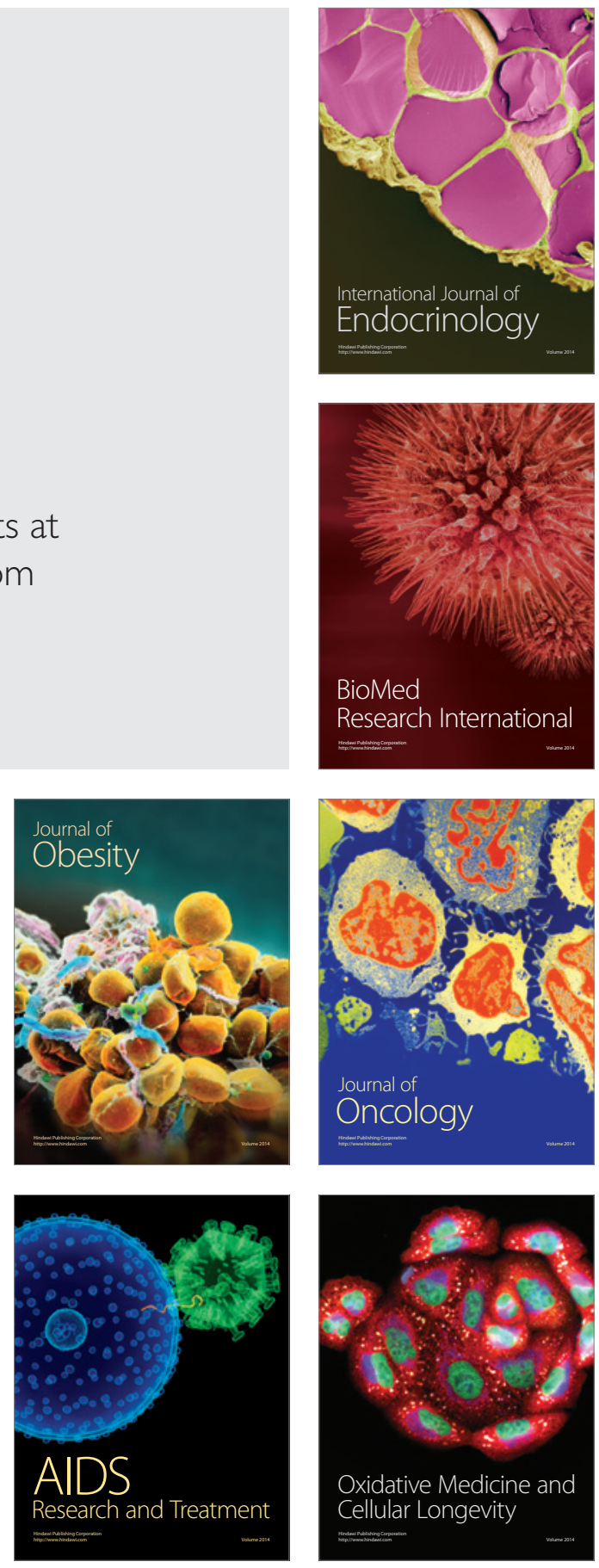